\title{
GLOBAL ASPECTS OF DYNAMICS AND STAR FORMATION IN TAURUS
}

\author{
ANa I. Gomez de CASTro ${ }^{1,2,3}$ and Ralph E. Pudritz ${ }^{1,4}$
}

Received 1991 May 24 ; accepted 1992 February 27

\begin{abstract}
We employ a number of data sets that include the proper motions of T-Tauri stars, molecular and $\mathrm{H}$ I velocities and densities, optical polarimetry, and $\mathrm{H} \alpha$ luminosities of young stars in order to derive a global view of gas dynamics and star formation in the Taurus molecular cloud. We find strong correlations among cloud properties. TTS stars move in a direction that is roughly parallel to the gaseous filaments in the cloud. Assuming that stars and gas are well coupled, we infer that the cloud moves through the ISM at $\sim 17 \mathrm{~km} \mathrm{~s}^{-1}$. The Taurus cloud is a member of Gould's Belt, and it is moving in the same direction as the other members.

We propose that the cloud's motion arises as a consequence of its formation through Parker instability in the ISM. The bulk of gas displaced by this instability moves laterally at several times the Alfvén speed of the undisturbed gas as it falls back toward the galaxy. It is this motion that is consistent with that of Taurus. An odd (antisymmetric with respect to reflection through the Galactic plane) mode Parker instability produces a warped gas layer which we interpret to be the origin of Gould's Belt, of which Taurus is a small constituent. The infalling gas will shock with material in the plane of the Belt. MHD waves are generated in this process, and we suggest that this is the origin of large-scale Alfvén waves in Taurus. Such waves do not damp for the life of the cloud and keep it supported against global gravitational collapse. The gravitational energy release during the Parker instability is sufficient to account for the kinematics of the cloud as well as its internal stirring.

The motion of the Taurus cloud is mildly super-Alfvénic with respect to the surrounding undisturbed WNM, and this must be associated with a shock. There are indications of the presence of a shock of speed $v_{\text {sh }} \simeq 6.7 \mathrm{~km} \mathrm{~s}^{-1}$ in Taurus. This shock is suggested to be responsible of the relative velocity drift of $3.8 \mathrm{~km}$ $\mathrm{s}^{-1}$ between the weaker $\mathrm{H}_{\mathrm{I}}$ self-absorption component and the rest of the cloud (CO, H I emission, PMS stars). Observational support for the existence of a large-scale Alfvén wave in Taurus comes from both the wavelike distortion of the cloud magnetic field (wavelength of $17 \mathrm{pc}$ ) and the wavelike disturbance in the velocity field of this $\mathrm{H}$ I component which matches up with the magnetic data.

Finally, the influence of the shock in the star formation process is analyzed. It is shown that there are no significant differences in the evolutionary state of groups of PMS stars located at very different positions in the cloud. It appears as if many features of the dynamics and physics of the cloud are determined by global issues such as the process of cloud formation and its interaction with the larger scale ISM.
\end{abstract}

Subject headings: interstellar: molecules - nebulae: individual (Taurus Dark Cloud) nebulae: internal motions - shock waves - stars: formation — stars: pre-main-sequence

\section{INTRODUCTION}

The Taurus molecular cloud is a very well studied region of star formation. Comprehensive mapping in various molecular probes such as $\mathrm{CO}$ and $\mathrm{NH}_{3}$, detailed studies of the young stellar objects and T-Tauri stars, and thorough studies of the cloud magnetic field by optical polarization techniques allow one to explore the relation between star formation and magnetic gas dynamics. Optical studies (Barnard 1927; Duvert, Cernicharo, \& Baudry 1986; Cernicharo, Bachiller, \& Duvert 1985) and CO maps (Ungerechts \& Thaddeus 1987, hereafter UT; Heyer et al. 1987) reveal that much of the cloud has a highly filamentary substructure in which dense $\mathrm{NH}_{3}$ cores are embedded (Myers \& Benson 1983; Benson \& Myers 1989). The geometry of the magnetic field in Taurus has been traced by optical polarization measurements (Vrba, Strom, \& Strom 1976; Moneti et al. 1985, Hsu 1984; Heyer et al. 1987;

\footnotetext{
${ }^{1}$ Department of Physics and Astronomy, McMaster University, Hamilton, Ontario, Canada L8S 4M1.

${ }^{2}$ NSERC International Postdoctoral Fellow.

3 Current address: IUE Observatory-VILSPA, P.O. Box 50727, 28080 Madrid, Spain.

${ }^{4}$ CITA, University of Toronto, Toronto.
}

Goodman et al. 1990). The observations indicate that the field is well ordered on the scale of the whole cloud, but that it also contains a significant disordered component on the smaller scales (Goodman et al. 1990).

Zeeman measurements of the field strength in molecular clouds indicate that magnetic and gravitational energy densities are comparable. Thus, magnetic fields must play a fundamental role in the dynamics of the cloud and the formation of structures such as the ubiquitous cores and filaments. The orientation of the magnetic field with respect to the dense filament substructures can vary from perpendicular to parallel alignments depending upon the filament (Vrba et al. 1976; Schwartz 1977; Heyer et al. 1987; Goodman et al. 1990). Within Taurus, one finds both of these orientations. Therefore, while the magnetic field must play an important role in the formation of cloud structure and star formation, the relative alignment of the magnetic field and cloud substructure is still somewhat mysterious.

In order to build up a more detailed idea of the global gas dynamics in the Taurus complex, velocities of the gas and young stars must be studied. Unfortunately one cannot obtain information about motion of the gas in the plane of the sky. However, the young stars within the cloud have measurable 
proper motions, and their radial velocities show that they are well coupled with the gas (Herbig 1978; Hartmann et al. 1986). Thus, one can use the velocities of the T-Tauri stars (TTS) in Taurus to build up a three-dimensional picture of cloud dynamics.

In this paper we combine the measured proper motions of TTS in Taurus with the observed properties of the gas and magnetic field in the cloud in order to deduce the global dynamics of the Taurus cloud. The results show that there are surprisingly strong correlations among these cloud properties. One of the most striking observational features is that TTS appear to move in a direction that is approximately parallel to the filaments. This motion and the location of the region indicates that Taurus is part of the Gould's Belt. The Gould's Belt is a massive kiloparsec-size region of $O B$ stars and molecular clouds in our nieghborhood that form an important starforming system. The global view suggests that some aspects of the internal dynamics of Taurus may be coupled with the motion of the cloud as a whole through the interstellar medium. In fact, the data support two interesting properties of the Taurus cloud, namely that (1) a large-scale Alfvén wave is propagating through it and (2) there is an MHD shock propagating through the region in a direction that is roughly perpendicular to the cloud field (i.e., the shock front is roughly parallel to the cloud field).

We show that these global aspects of the Taurus cloud dynamics and morphology can be accounted for if the cloud was produced as a consequence of a Parker-Jeans instability. This instability is generally thought to be the mechanism by which molecular clouds form (e.g., Elmegreen 1982; Blitz \& Shu 1980). The energetically most favored Parker unstable mode for galactic gas in the presence of a vertically varying gravitational acceleration is antisymmetric with respect to reflection through the Galactic plane (Horiuchi et al. 1988). The infall of gas during the operation of such an odd-mode Parker instability can produce a copious flux of Alfvén waves (Matsumoto et al. 1988), and we suggest that this is the origin of the wavelike disturbances observed in the $\mathrm{H} \mathrm{I} 21 \mathrm{~cm}$ data of

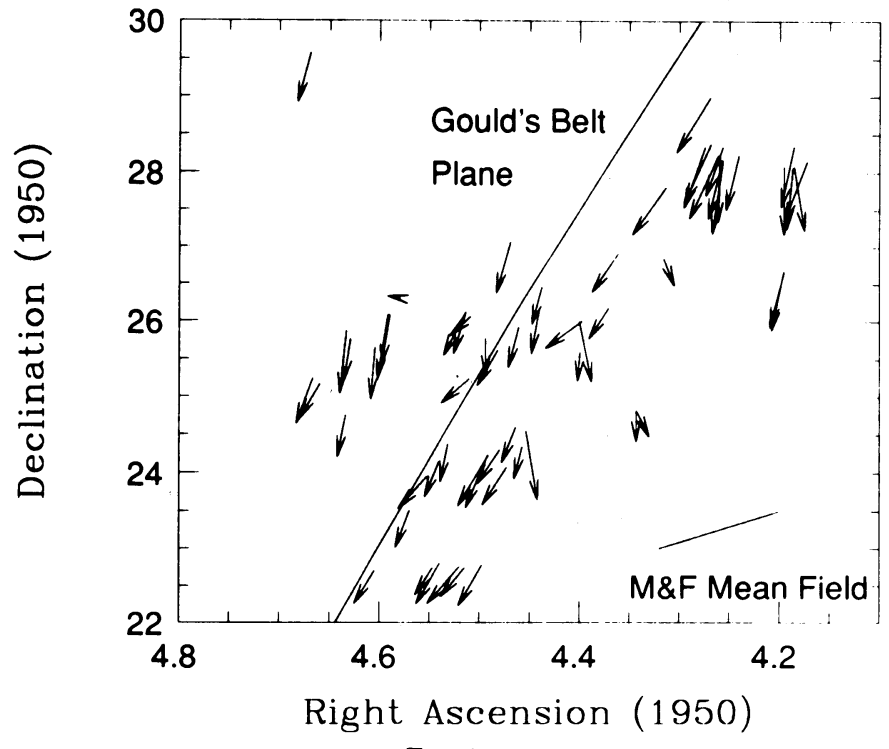

FIG. $1 a$
Shuter, Dickman, \& Klatt (1987) as well as the optical polarization data. Gas that is displaced by the Parker instability moves at super-Alvénic speeds and therefore shocks with the surrounding undisturbed ISM. We show that there are good reasons to suspect that an MHD shock wave is propagating in Taurus.

We present the stellar proper motion data and a wide variety of gas and magnetic data and infer the gas velocity field and the three-dimensional magnetic geometry in the next section $(\$ 2)$. We examine the link between the Taurus cloud and the larger scale interstellar medium in $\S 3$. We then present a unifying model for understanding the global features of the cloud in terms of the Parker-Jeans instability picture for molecular cloud formation $(\S 4)$. Such a picture predicts that waves and shocks should be produced within the cloud, and we examine these issues in $\S \S 5$ and 6 respectively. Finally, we discuss the links between the global dynamics of the Taurus cloud and the star formation within it $(\S 7)$.

\section{CLOUD DYNAMICS AND MAGNETIC FIELD GEOMETRY}

\subsection{TTS Velocity Field}

We collected data on the proper motions of 59 TTS from Jones \& Herbig (1979, hereafter JH) as well as four new TTS found by Hartmann et al. (1991). In Figure $1 a$ we plot the proper motion vectors on the scale of the Taurus cloud. In Figure $1 b$ we reproduce, for comparison, the IRAS map of Taurus with its optical polarization vectors, as compiled by Scalo (1990). There is a strong streaming effect seen in these proper motions. The stars defined by JH as group I are concentrated at the NW end of the complex and are associated with the L1495 and P1454 dark clouds. Their velocity, in the mean, is $5.7 \mathrm{~km} \mathrm{~s}^{-1}$ larger than that of the TTS in the center of the

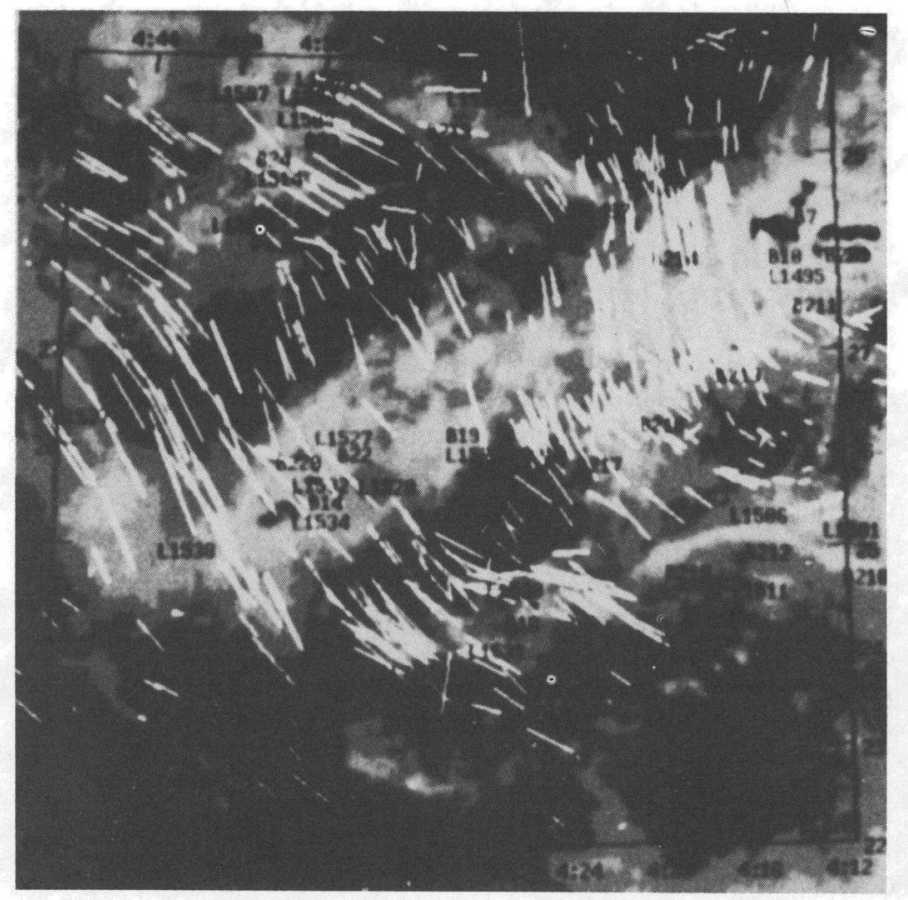

FIG. $1 b$

Fig. 1. $-(a)$ Proper motions of the TTS in Taurus. The orientation of the large-scale mean magnetic field as defined by Mathewson \& Ford (1970) data is indicated in the lower right corner of the figure. The location of the Gould's Belt plane is also indicated. (b) The polarization map of Taurus compiled by Scalo (1990). 
cloud (JH's group II). This fact motivated JH to suggest that both groups could be dynamically different.

The radial velocities are known only for 31 of the TTS located in the region mapped in Figure 1 (Herbig 1978; Hartmann et al. 1986). In the mean, the measured radial velocity respect to the LSR is $6.5 \mathrm{~km} \mathrm{~s}^{-1}$.

The velocity in the plane of the sky of group I stars is 18.4 $\mathrm{km} \mathrm{s}^{-1}$ while that in group II is $13.6 \mathrm{~km} \mathrm{~s}^{-1}$. The mean P.A. of the proper motion vectors of the combined sample of all the stars is

$$
\langle\text { P.A. }\rangle_{\text {prop }} \simeq 164^{\circ} \text {. }
$$

The magnitudes of the TTS total velocities are $\sim 19.5 \mathrm{~km}$ $\mathrm{s}^{-1}$ and $\sim 15.1 \mathrm{~km} \mathrm{~s}^{-1}$, respectively. These results imply that the inclinations of the velocity vectors respect to the line of sight are

$$
\begin{aligned}
i=\tan ^{-1}\left(v_{\text {prop }} / v_{\text {rad }}\right) & \simeq 71^{\circ} \quad(\text { Group I }) \\
& \simeq 65^{\circ} \quad(\text { Group II }) .
\end{aligned}
$$

Taking the average of the motions of all the TTS stars and assuming that they are frozen into the motion of the gas in which they formed (justified in the next subsection), these results indicate that the cloud is moving at a velocity of $\simeq 16.5$ $\mathrm{km} \mathrm{s}^{-1}$ with respect to Local Standard Rest with a very small component in the line-of-sight direction (inclination, $i \simeq 67^{\circ}$ ). It appears therefore that the TTS, and therefore the Taurus cloud, are moving predominantly in the plane of the sky.

The intrinsic proper motion dispersions were also calculated by $\mathrm{JH}$. The values could not be estimated for individual groups because, in general, the error dispersions were of the order of the observed dispersions. The main conclusions from the JH analysis were (1) the intrinsic velocity dispersions for all the stars in the Taurus association are $3.2 \pm 1.3 \mathrm{~km} \mathrm{~s}^{-1}$ and $2.2 \pm 1.6 \mathrm{~km} \mathrm{~s}^{-1}$ in the directions of right ascension and declination, respectively, (2) the stars in tight concentrations show smaller velocity dispersions, and (3) the stars in group I show a velocity anisotropy with a dispersion in right ascension about twice than in declination $\left(0.53\right.$ cent $^{-1}$ versus 0.25 cent $\left.^{-1}\right)$.

\subsection{Coupling of Stars and Gas}

There is no evidence of any systematic difference between the mean velocity of the stars and that of the molecular cloud. Moreover the radial velocity dispersion of the stars with respect to the gas is smaller than $1.5 \mathrm{~km} \mathrm{~s}^{-1}$ (Herbig 1978; Hartmann et al. 1986) which is consistent with the internal motions of the gas (see UT). The velocity dispersion in one coordinate inferred from the proper motions is typically $2-3$ $\mathrm{km} \mathrm{s}^{-1}$, and smaller than the escape velocity from the clouds (JH). Therefore if clouds and stellar motions are as well coupled as these data suggest, then the filaments that constitute the Taurus clouds are moving in the direction of the TTS proper motions.

Further indications of the coupling between the molecular gas in the cloud and the stars come from the velocity gradient along the cloud which is $0.062 \mathrm{~km} \mathrm{~s}^{-1} \mathrm{pc}^{-1}$. Kinematic studies of the molecular gas show that there is a well-defined velocity gradient in the NW-SE direction along the cloud (UT). This gradient cannot be due to galactic differential rotation, and the motion seems to be linked to the peculiar kinematics of the Taurus cloud itself.

The gradient is also apparent in the stellar component when comparing the mean radial velocities of the stars in groups I and II. We have estimated these values from the radial velocities given by Hartmann et al. (1986), on the basis of only seven measured stars in region I and nine in region II. The mean radial velocities and dispersions are:

$$
\begin{array}{ll}
V_{r}=7.4 \pm 2 \mathrm{~km} \mathrm{~s}^{-1} & (\text { Region I) } \\
V_{r}=6.3 \pm 1.5 \mathrm{~km} \mathrm{~s}^{-1} & \text { (Region II) }
\end{array}
$$

Thus the stellar gradient is approximately of order $0.085 \mathrm{~km}$ $\mathrm{s}^{-1} \mathrm{pc}^{-1}$, and the velocity dispersions are comparable between the two groups and the cloud as a whole.

\subsection{Filament Morphology}

Taurus filaments share an interesting morphology with filaments in other molecular clouds in that they appear to have a cometary shape. In Taurus, the "head" of the comet points in approximately the same direction as the TTS proper motions. The densest clumps, the $\mathrm{NH}_{3}$ cores, and the PMS stars are mainly concentrated at the head positions. L1495 and P1454 are at the head of the filament that connects (in projection) Taurus with Perseus (see UT CO map). B217 is at the SE end of filament GF 13 (Scheneider \& Elmegreen 1979), and TMC2 and B18 also at the SE end of GF 16. The same phenomenon is observed in Heiles Cloud 2 (HCL2). Therefore the filaments seem to know about the direction marked by the motion of TTS.

As an aside we note that chemical gradients also seem to follow the directions of the filaments. A good example is the strong gradient in the $\mathrm{NH}_{3}$ /cyanopolyne abundance ratio that has been observed over a distance of $0.2 \mathrm{pc}$ in this same direction in the HCL2-A clump (Cernicharo, Guélin, \& Askne 1984a; Cernicharo et al. 1984b).

\subsection{H I Gas Associated with Taurus}

Shuter et al. (1987) mapped a $10^{\circ} \times 7^{\circ} .5$ region in the center of Taurus, and found a $21 \mathrm{~cm}$ self-absorption feature with a systemic velocity of $2.7 \mathrm{~km} \mathrm{~s}^{-1}$. A detailed correlation study of this $\mathrm{H}$ I velocity field shows that there is a significant correlation with a length scale of

$$
\lambda_{\mathrm{HI}} \simeq 16 \mathrm{pc} \text {. }
$$

These authors argue that this is a velocity wave propagating through the complex. The direction of propagation of the wave projected in the plane of the sky is

$$
\langle P A\rangle_{\mathrm{H} \mathrm{I}} \simeq 48^{\circ}
$$

measured in Figure $2 c$ of Shuter et al. (1987). A rather significant point is that both the ${ }^{13} \mathrm{CO}$ and the $\mathrm{H}$ I emission mapped at similar points have the same systemic velocity of $v_{\mathrm{CO}}=6.5$ $\mathrm{km} \mathrm{s}^{-1}$. However, the $\mathrm{H}$ I gas seen in absorption has a rather different speed, being

$$
v_{\mathrm{CO}}-v_{\mathrm{H} \mathrm{I}, \text { abs }} \simeq 3.8 \mathrm{~km} \mathrm{~s}^{-1} \text {. }
$$

This velocity difference is difficult to explain, and the authors proposed that passage of the molecular complex through ambient $\mathrm{H}$ I gas would produce a wake in the $\mathrm{H}$ I which could be seen in absorption. The shape of the $\mathrm{H}_{\mathrm{I}}$ isocontour maps clearly indicates that the self absorption is associated to the Taurus cloud (Shuter et al. 1987).

Shuter \& Dickman (1989) also noted the existence of a correlation length in the column density of the $\mathrm{H}$ I of $\sim 11 \mathrm{pc}$. This is smaller than the $\sim 16 \mathrm{pc}$ correlation length of the velocity field, and we return to address this issue later in the paper.

The layer that is producing the $\mathrm{H}$ I self-absorption has a very 
low column density. A gross estimate of the column derived by us from the $\mathrm{H}_{\mathrm{I}} 21 \mathrm{~cm}$ line profile published by Shuter et al. (1987) gives

$$
\log _{10} N(\mathrm{H} \mathrm{I})\left(\mathrm{cm}^{-2}\right) \leq 19.0 \text {. }
$$

This value is probably already overestimated by an order of magnitude (Wilson \& Minn 1977).

\subsection{The Cloud Magnetic Field Inferred from Optical Polarization}

The magnetic field delineated by optical polarization vectors of background stars is shown in Figure 1 (compiled in Scalo 1990). The vectors are taken from Hsu (1984), Moneti et al. (1985), and Heyer et al. (1987). The data include only vectors whose position angle uncertainty is less than $5^{\circ}$. The field geometry clearly changes across the cloud. The general trend is that of a wavelike ripple with a characteristic scale of

$$
\lambda_{\mathrm{pol}} \simeq 17 \mathrm{pc}
$$

extending from the NE to the SW with P.A. $\simeq 50^{\circ}$. It is interesting that the P.A. of this ripple is essentially that observed in the $\mathrm{H}$ I wave, which we discussed above. A change in the orientation of the field across the cloud has also been claimed by Vardanyan \& Eritsyan (1984) from the study of the isodeclination line RY Tau-T Tau, where $\alpha(1950) \simeq 4^{\mathrm{h}} 20^{\mathrm{m}}$ and the declination varies between $\delta(1950) \simeq 19.4$ and 28.3 .

It is striking that $\lambda_{\mathrm{HI}} \simeq \lambda_{\text {pol }}$. In fact, a glance at the $\mathrm{H} \mathrm{I}$ velocity map given in Figure $2 b$ of Shuter et al. (1987) shows good agreement with the geometry inferred from the polarization map. The $\mathrm{H}$ I velocity field has essentially three peaks, two at $V=1 \mathrm{~km} \mathrm{~s}^{-1}$ with respect to the systemic velocity of the $\mathrm{H}_{\mathrm{I}}$ self-absorption, that are located at $\left(\alpha_{1}, \delta_{1}\right) \simeq\left(4^{\mathrm{h}} 42^{\mathrm{m}}, 28^{\circ} 30^{\prime}\right)$ and $\left(\alpha_{2}, \delta_{2}\right) \simeq\left(4^{\mathrm{h}} 17^{\mathrm{m}}, 25^{\circ} 30^{\prime}\right)$ and another at $V=-1 \mathrm{~km} \mathrm{~s}^{-1}$ placed over the center of the Taurus cloud $\left(\alpha_{3}, \delta_{3}\right) \simeq\left(4^{\mathrm{h}} 27^{\mathrm{m}}\right.$, $26^{\circ}$ ). The three peaks are aligned in the direction P.A. $\sim 48^{\circ}$, and the correlation length estimated by Shuter et al. (1987) is approximately the distance between the two maxima at $V=1$ $\mathrm{km} \mathrm{s}^{-1}$.

The most striking aspect of the data plotted in Figure 1 is that the TTS move in a direction that is approximately parallel to the filaments and nearly perpendicular to the cloud field direction. The strongest variations of the orientation of the polarization vectors are observed in the northwest associated with L1495 and P1454 clouds. This is the region of the Taurus complex where the column density of CO is larger (see UT).

A recent quantitative analysis of the Taurus polarization (Myers \& Goodman 1991) models the cloud field as consisting of uniform and nonuniform parts. The nonuniform component is isotropic in two dimensions for a wavelike case or three dimensions for a turbulent case and has a Gaussian distribution of amplitude. Using a sample of over 300 polarization measurements, these authors find that the P.A. for the uniform field component $B_{0}$ is

$$
\langle\text { P.A. }\rangle_{B_{0}}=48^{\circ} \pm 1
$$

with a dispersion of $25^{\circ} \pm 0.6 \mathrm{rad}$. Note that this range of angles is consistent with the amplitude of the wavelike ripple we have discussed. The mean field direction is quite different than the larger scale $(1 \mathrm{kpc})$ galactic field mapped and modeled by Mathewson \& Ford (1970) and Mathewson (1968) whose P.A. is $\sim 109^{\circ}$.

An important feature of this mean field direction
( $\langle\text { P.A. }\rangle_{B_{0}}=48^{\circ}$ ) is that it precisely lines up with the P.A. of the wave propagation direction in the Shuter et al. (1987) data.

\subsection{Three-dimensional Magnetic Field in Taurus}

The line-of-sight component of the magnetic field $B_{\|}$can be determined by detecting the Zeeman splitting of the $21 \mathrm{~cm}$ line. Only an upper limit to $B_{\|}$has been established from $21 \mathrm{~cm} \mathrm{H} \mathrm{I}$ line measurements, and it implies a very small field component of $B_{\|} \leq 5 \mu \mathrm{G}$ (Verschuur 1971). At first glance this is surprising since magnetic virial theorem arguments suggest that the minimum strength $B_{c}$ that will support a spherical cloud against collapse in directions perpendicular to the field is

$$
B_{c}=30\left(M / 10^{4} M_{\odot}\right)^{1 / 2}\left(\rho / 10^{-21} \mathrm{~g} \mathrm{~cm}^{-3}\right) \mu \mathrm{G},
$$

which implies a field strength for Taurus of $B_{c}=12 \mu \mathrm{G}$. The estimated field intensity in equation (2.2) is deduced assuming the total mass of the Taurus Complex given by UT from CO molecular maps: $2.26 \times 10^{4} M_{\odot}$, and that a representative column density (from the same work) is $4 \times 10^{21} \mathrm{~cm}^{-2}$. Thus for a representative cloud thickness of $\sim 10 \mathrm{pc}$, the mean density in the cloud is $n=130 \mathrm{~cm}^{-3}$ and $\rho=2.6 \times 10^{-22} \mathrm{~g}$ $\mathrm{cm}^{-3}$, and therefore a cloud field strength of $\sim 12 \mu \mathrm{G}$ is expected. Recent $\mathrm{OH}$ Zeeman measurements have put an upper limit of $18 \mu \mathrm{G}$ on any magnetic field in the core Tau 16 (Goodman et al. 1989), and this tends to support our estimate. Indeed, direct measurements of field strengths in many molecular clouds show that they are relatively close to magnetic virial equilibrium (Myers \& Goodman 1988).

This puzzle in Taurus can be resolved if the field direction is mainly parallel to the plane of the sky. We can constrain the orientation of the Taurus field in space by assuming that the total field is comparable in strength to that predicted by the virial theorem, an assumption which we know is fully justified for most other clouds. Thus if the total field strength is $B_{t} \simeq$ $B_{c} \simeq 12 \mu \mathrm{G}$, then it has an inclination $i_{B}$ with respect to the line of sight,

$$
i_{B}=\cos ^{-1}\left(B_{\|} / B_{t}\right) \simeq 65^{\circ} .4 .
$$

Our estimate is based on the virial argument which has been similarly used by Goodman et al. (1990).

The foregoing estimates of the field strength pertain to dense atomic gas of density of $300 \mathrm{~cm}^{-3}$ (Troland \& Heiles 1982). The mean Alfvén speed in the cloud is therefore

$$
V_{\mathrm{A}} \leq 1.4 \mathrm{~km} \mathrm{~s}^{-1} \text {. }
$$

This is approximately the line width of the CO lines in the main cores of the complex (Myers, Linke, \& Benson 1983; $\Delta v \leq 1.5 \mathrm{~km} \mathrm{~s}^{-1}$ in ${ }^{13} \mathrm{CO}$ ), and the field is also of the order observed in other low-mass star-forming regions such as L204 (Myers \& Goodman 1988; Heiles 1988). The implicit argument underlying this is the often assumed scaling $\delta v \simeq V_{\mathrm{A}}$.

\subsection{Relation Between Field Geometry and Cloud Dynamics}

Since the ambient field is relatively well frozen into the molecular gas and the Taurus complex is itself streaming at 17 $\mathrm{km} \mathrm{s}^{-1}$ with respect to the galactic corotation speed at Taurus galactocentric distance, we come to the question of why the Taurus field has a rippled geometry.

Imagine first that the cloud field connects with the external ISM (in principle, at rest with respect to the LSR) immediately 
at the cloud boundary. The field would significantly distort whenever the cloud's motion through the surrounding quiescent medium exceeds the Alfvén speed. Since the latter is only $1.5 \mathrm{~km} \mathrm{~s}^{-1}$, the field lines would be expected to drape around the Taurus cloud making an angle $\theta=\tan ^{-1}\left(1.5 \mathrm{~km} \mathrm{~s}^{-1} /\right.$ $\left.17 \mathrm{~km} \mathrm{~s}^{-1}\right)=5^{\circ}$ between the streaming direction and that of the field. The streaming direction has a P.A. in the plane of the sky, $\langle\text { P.A. }\rangle_{\text {stream }} \simeq 163^{\circ}$, and the minimum and maximum P.A. of the Taurus magnetic field are $-15^{\circ}\left(165^{\circ}\right)$ and $125^{\circ}$ (Goodman et al. 1990). This would imply that in the southwest part of the cloud the field is essentially parallel to the streaming direction (see Fig. 1) and compatible with the distortion expected if connected with the ISM field. However, in the northeast, the field forms an angle of, at least, $38^{\circ}$ with the streaming direction that cannot be interpreted this way.

The simplest explanation of the data is that most of the polarization measurements trace a magnetic field that is either internal to the cloud (and hence moves with it), or is associated with gas that moves as the cloud moves.

\subsection{Summary}

The analysis of the gas dynamics and field geometry reveals that (1) the wavelike ripple in the magnetic field is also seen in the $\mathrm{H}$ I self-absorption velocity field; (2) the cloud (CO and $\mathrm{H} \mathrm{I}$ emission) and the $\mathrm{H}$ I self-absorption move at different speeds; (3) stellar motions are roughly parallel to the filaments and the Gould's Belt plane and indicate the direction of motion of the Taurus cloud; and (4) the rippled field is oriented roughly perpendicular to the filaments and is moving together with the cloud at $16 \mathrm{~km} \mathrm{~s}^{-1}$ respect to the local interstellar medium.

These results require that we examine the larger scale ISM and Taurus's interaction with it. We turn to this larger scale next.

\section{TAURUS AS A MEMBER OF GOULD'S BELT}

An important link of Taurus to the larger scale ISM is its membership in the dynamical entity known as Gould's Belt. The Gould's Belt traces the distribution of young mainsequence stars (OB), $\mathrm{H}$ II regions, and dark clouds in a radius of $\sim 500 \mathrm{pc}$ around the Sun. All these objects lie in a plane inclined $\sim 18^{\circ}$ with respect to the Galaxy and participate in the same kinematics (Stothers \& Frogel 1974; Frogel \& Stothers 1977). For future convenience let us note that the position angles of Gould's Belt and the Galactic plane toward Taurus are

$$
\begin{aligned}
\langle P A\rangle_{\mathrm{G}} & \simeq 150^{\circ}, \\
\langle P A\rangle_{\mathrm{Gal}} & \simeq 140^{\circ},
\end{aligned}
$$

respectively. The radial velocity of the Taurus cloud is typical for the molecular clouds in the Gould's Belt system as shown by Taylor, Dickman, \& Scoville (1987; see Fig. 5 in their paper). The three components of the spatial motion of the TTS indicate that they are moving in the same direction as the rest of the Gould's Belt stars although the total space motion is some $10 \mathrm{~km} \mathrm{~s}^{-1}$ slower (Eggen 1986, 1989).

The origin of the motion of clouds and stars within Gould's Belt is still unknown. It has been modeled as an expansion produced by supernova explosions with kinematical times of the order of $4.5 \times 10^{7} \mathrm{yr}$ (Lesh 1968), 2-6 $\times 10^{7} \mathrm{yr}$ (Frogel \& Stothers 1977). However, detailed studies of the kinematics of OB stars in the Belt (see, e.g., Westin 1987) find that such a supernova model has some difficulty in explaining the observed motions. A more obvious difficulty with the supernova scenario is that there is no explanation for the observed tilt of the entire Belt system, both in the stars and gas. The Parker-Jeans instability model for the origin of Gould's Belt that we propose in the next section alleviates these difficulties, but more details must await another paper.

The belt thickness has been determined both for the molecular clouds and the stars. The characteristic scale height of the OB stars in Gould's Belt (Stothers \& Frogel 1974) is about 17 pc. The upper limit to the scale height of the molecular clouds is $30 \mathrm{pc}$ (Taylor et al. 1987).

\section{CONSEQUENCES OF THE PARKER-JEANS INSTABILITY}

It is generally thought that the formation of molecular clouds begins with the onset of a Parker instability in the magnetic field of the galaxy (e.g., Blitz \& Shu 1980; Elmegreen 1982). In the standard view, a passing spiral density wave compresses a magnetized section of the galaxy, and the predominantly azimuthal field becomes buoyant and bubbles out of the disk. The gas is raised and pushed to the side in such a motion. Ultimately it falls back toward the Galactic plane. The newly formed gas concentrations in the plane become selfgravitating, and molecular clouds are formed.

Elmegreen (1982) has investigated the evolution of the Parker instability including the effects of self-gravity in the linear regime. He finds that the instability time scale can be much less than the free-fall time perpendicular to the plane if the galactic gas density exceeds $\simeq 5 \mathrm{~cm}^{-3}$. His calculations show that

$$
t_{\text {inst }}=\frac{H}{\eta u} \simeq 2.24 \times 10^{7} \eta^{-1} \mathrm{yr}
$$

where $\eta$ is a numerical factor of $O(1)$, and $u \simeq 7 \mathrm{~km} \mathrm{~s}^{-1}$ is the one-dimensional $\mathrm{rms}$ speed in the interstellar medium. For the stated conditions, growth rates of $1.2 \times 10^{7} \mathrm{yr}$ are found.

The energetics of the gas motion during the instability are easily generalized from the simple argument of Matsumoto et al. (1988). Initially we imagine a layer with scale height $H_{i}=$ $(1+\alpha) c_{s}^{2} / g_{i}$, where $g_{i}$ is the gravitational acceleration, $c_{s}$ is the gas sound speed, and $\alpha \equiv B^{2} / 8 \pi \rho_{i} c_{s}^{2}$. After the instability has taken place, the magnetic field has buoyed up out of the gas. The gas ultimately settles to the reduced scale height, $H_{f}=$ $c_{s}^{2} / g_{f}$. The speed of the displaced gas is determined from the gravitational energy released, namely $\left(\frac{1}{2}\right) \rho_{f} v_{f}^{2}=\rho_{i} g_{i} H_{i}$ $-\rho_{f} g_{f} H_{f}$. Thus the bulk motion of the gas calculated in this way is

$$
v_{f}=2^{1 / 2}\left[\left(\rho_{i} / \rho_{f}\right)(1+\alpha)-1\right]^{1 / 2} c_{s} .
$$

In the limit at which there is little density constrast between the initial and final gas layer, then $v_{f} \simeq v_{\mathrm{A}}$, where $v_{\mathrm{A}}$ is the Alvén speed in the undisturbed layer. Thus one reaches the conclusion that gas should be moving at speeds comparable to the Alfvén speed in the undisturbed gas. More accurate results depend on nonlinear simulations, and Matsumoto et al. (1988) find lateral and vertical gas motions with speeds of order

$$
\begin{aligned}
& v_{x} / v_{\mathrm{A}} \simeq 1.3 \\
& v_{z} / v_{\mathrm{A}} \simeq 1.9 .
\end{aligned}
$$

Shocks develop if $\alpha \geq 1$.

Most discussions of the Parker instability emphasize the linearized physics of an even mode, that is, one whose magnetic geometry is mirror symmetric with respect to reflection 


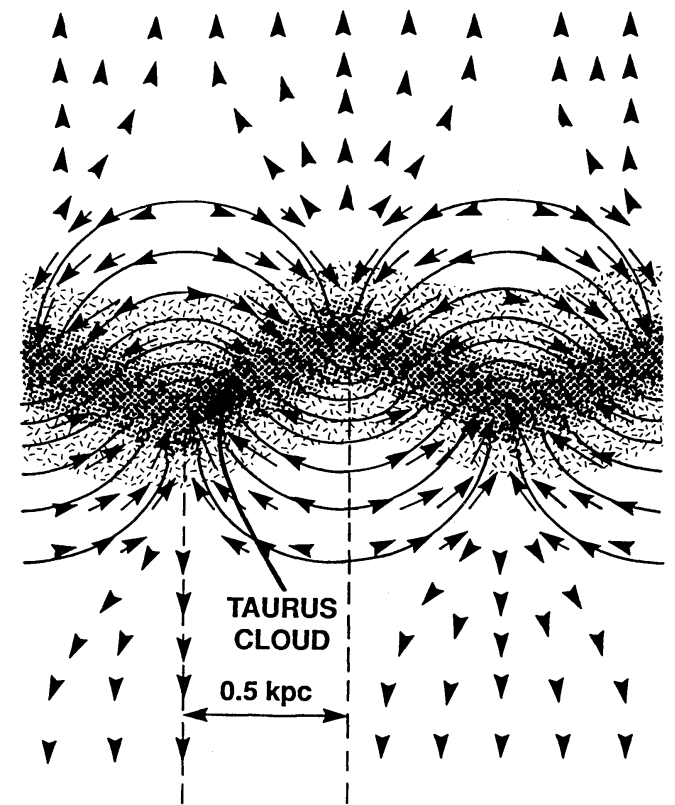

FIG. 2.-Sketch of the mechanism to generate a large-scale Alfvén wave in Taurus through an odd-mode, Jeans-Parker Instability. The figure is adapted from detailed numerical simulations of Matsumoto et al. (1988). The arrows represent the velocity vectors of gas displaced by the buoyant magnetic field. The gas rains back down to the Galactic plane along these magnetic field lines (solid lines). The field in the shocked gas layer generates the observed Alfvén waves. Residual motion parallel to the plane can explain the motion of Taurus cloud in the Gould's Belt plane. An odd-mode instability produces a warped plane as shown.

through the Galactic plane. While most of the literature addresses situations in which the vertical gravitational acceleration $g$ is a constant, Horiuchi et al. (1988) have examined the case where $g$ varies with height $z$. In this case, a discrete spectrum of modes is found. The lowest energy mode is one which is antisymmetric with respect to reflection through the Galactic plane; that is, the magnetic structure below the plane is found by reflecting the structure above the plane and then shifting it by half a wavelength. This mode is illustrated in Figure 2.

This type of mode is important because the displaced gas ends up on a sheet that is inclined with respect to the Galactic plane. Thus, the instability leads to a warped disk. This is fundamental to understanding the origin of a structure such as Gould's Belt which is significantly inclined in precisely this way. Matsumoto et al. (1988) have studied the nonlinear development of such modes numerically. We have adapted a figure from their paper and present it in Figure 2. Their calculations show that during the nonlinear development of the Parker instability, gas flowing down the field lines back toward the Galactic plane collides and shocks with the material already deposited in the layer. The arrows in Figure 2 mark the sense of the velocity field in their simulations. We have emphasized that a layer, inclined with respect to the Galactic plane, will form and that this is essentially Gould's Belt.

A copious flux of Alfvén waves is produced in this formation process. The reason for this is understood by closely examining Figure 2. The infall associated with an odd-mode Parker instability produces a sheared gas layer since gas arriving from above and below the tilted shocked layer is of opposite direction. This relative shear is transverse to the layer, and Alfvén waves set up on the threading magnetic field. Thus wave generation is an integral part of molecular cloud formation in the
Gould's Belt context. The point is that Alfvén wave production is intrinsic to the formation of molecular clouds. This gives an explanation for the ubiquitousness of powerful hydromagnetic wave fluxes within molecular clouds, in general, and Taurus in particular.

Finally, we emphasize that the motion of the gas in the shocked gas layer takes on the character of an expanding system of clouds, as the observations of Gould's Belt system suggest. The reason for this is just that the component of momentum of the gas in a direction parallel to the layer $\left(v_{x}\right)$ is not canceled out in the shock. The infall into the shocked layer is along magnetic field lines of the buoyed-up field. If this field line makes an angle of $\theta_{\mathrm{G}}$ with the normal to the Gould's Belt plane, then while the normal component of the momentum is nearly canceled in the shock, a transverse component survives. We interpret this as the velocity of the Taurus cloud in the Gould's Belt plane, $v_{\mathrm{G}} \simeq 17 \mathrm{~km} \mathrm{~s}^{-1}$.

The Taurus cloud velocity can be explained in terms of a Parker instability picture. We must first identify a plausible component of the instellar H I gas as the background. A natural choice is the WNM component which has a temperature of order $8000^{\circ}$. The velocity dispersion associated with this component is therefore $c_{s} \simeq 9 \mathrm{~km} \mathrm{~s}^{-1}$. If $\rho_{i} \simeq \rho_{f}$, and if the initial gaseous layer has equipartition between magnetic and thermal energy $(\alpha \simeq 1)$, then the simple theory in equation (4.2) predicts that $v_{f} \simeq 13 \mathrm{~km} \mathrm{~s}^{-1}$. Applying the results of the numerical simulations summarized in equation (4.3), one expects bulk motions of the resulting clouds of up to $17 \mathrm{~km} \mathrm{~s}^{-1}$ in accord with the observations.

The Taurus cloud shocks with the undisturbed ISM surrounding the Gould's Belt as it moves in the plane. Thus we are left with two major predictions from this odd-mode, Parker picture of cloud formation illustrated in Figure 2:

1. Transverse waves with wavelengths comparable to the sheared layer thickness can be generated, and

2. The cloud is left with a large component of velocity in Gould's Belt plane induced by the shock of the infalling material. Moreover the large velocity component in Gould's Plane might produce a shock of the cloud with the surrounding undisturbed ISM.

We now investigate these two major predictions of this idea in some detail.

\section{LARGE-SCALE HYDROMAGNETIC WAVES IN TAURUS}

The predominant field threading the Taurus cloud is, as we have seen, more or less perpendicular to the Gould's Belt plane. Disturbances in this field will propagate as Alfvénic waves. Much theoretical work starting from the important paper of Arons \& Max (1975) has established that such waves in a strong field stir up the largest scales in the cloud and thereby provide support against rapid global collapse. Because rotation and thermal pressure have energy densities that are far too small to provide support of the largest scales in the cloud against global collapse, only magnetic support through hydromagnetic waves is available. Since the wave power must be present on the largest cloud scales, the wave hypothesis predicts that one must detect hydromagnetic waves with wavelengths comparable to the cloud scale. The optical polarization data may indeed be showing up the existence of the requisite waves. In general, one should expect that a spectrum of hydromagnetic waves will be present; the stability of clouds stirred in 
this way has been examined in detail (Bonazzola et al. 1987; Pudritz 1990).

While it is not yet completely settled from the data, we argue that there is enough evidence to support the notion that the wavelike ripple can be in fact, a large-scale Alfvén wave. This, as we have already seen, is in agreement with the $\mathrm{H}$ I data as argued by Shuter et al. (1987). In the following we explore the consequences that the propagation of a large-scale wave has upon the physics of the cloud.

\subsection{Basic Characteristics of Large-Scale Waves}

First, let us determine the period of the wave from the apparent wavelength and Alfvén speed using the dispersion relation

$$
\omega=k V_{\mathrm{A}}
$$

where $k$ is the wave number and $\omega$ the wave angular velocity. The time scale inferred from this,

$$
T_{w}=(17 \mathrm{pc}) /\left(1.5 \mathrm{~km} \mathrm{~s}^{-1}\right) \simeq 1.1 \times 10^{7} \mathrm{yr},
$$

is a lower limit to the duration of the wave motion. The following subsection shows that waves of such long periods propagate virtually without damping for the life of the cloud. They are thus available for stirring the cloud up and providing support against gravitational collapse. The period of the inferred wave in Taurus is in good agreement with the ages of the TTS, and it is $50 \%$ smaller than the dynamical time estimated for the Gould's Belt $2 \times 10^{7}$ yr (e.g., Frogel \& Stothers 1977).

A period of $\simeq 10^{7} \mathrm{yr}$ for the wave motion is not fortuitous however. It is roughly the free-fall time of atomic gas over one scale height of the Galactic disk,

$$
t_{\mathrm{ff}}=\frac{\pi}{2}\left[\frac{H}{g(H)}\right]^{1 / 2}=1.9 \times 10^{7} \mathrm{yr},
$$

where the scale height $H=160 \mathrm{pc}$ and the gravitational acceleration is $g=3.5 \times 10^{-9} \mathrm{~cm} \mathrm{~s}^{-2}$ (Elmegreen 1982). The wave and dynamical time scales are therefore comparable, $T_{w} \simeq t_{\mathrm{ff}}$. Thus the stirring of Taurus and, indeed, of any molecular cloud may arise during its formation. The largest scale modes are the ringing fossils of that event.

Another important consequence of this physics is that the gravitational energy that is released as a consequence of the Jeans-Parker instability is ultimately responsible for the velocity dispersion of the cloud. If $\rho_{0}$ is the mean density of the infalling gas, $\rho$ the mean density of the molecular cloud that is formed, and $\delta v$ its internal velocity dispersion, then we expect that a good fraction of the energy in the infall will be converted into stirred postshock gas motions so that

$$
\delta v \simeq\left(\frac{\rho_{0}}{\rho}\right)^{1 / 2} v_{f, \text { tot }} \simeq 1.07 \mathrm{~km} \mathrm{~s}^{-1},
$$

where we take the $v_{f, \text { tot }} \simeq 2^{1 / 2} v_{f} \simeq 24 \mathrm{~km} \mathrm{~s}^{-1}$, and the density contrast between the infalling gas (taken to be roughly the same as the WNM) and the bulk molecular cloud to be $\rho / \rho_{0}=$ 500 .

The previous simple deduction raises the issue of whether or not a shock results preferrentially in mechanical wave production or heating. This question has been examined by Cox (1979) who finds that mechanical energy input into a cloud in the form of waves can be the most important method of putting energy into an interstellar cloud undergoing a shock.
Ultimately these waves damp out and heat the gas, as we shall see next.

\subsection{Wave Damping}

In this section, we address the question of what happens to waves that have been excited and that propagate through the bulk of the cloud in the postshock gas. The physics of wave propagation in molecular clouds has been recently extensively discussed (e.g., Pudritz 1990; Carlberg \& Pudritz 1990, hereafter CP). An essential point is that not all physical scales in a molecular cloud can be supported by the pressure of hydromagnetic waves. Waves of short enough wavelength damp by ion-neutral friction.

Alfvén waves with angular velocities larger than $2 \omega_{n, i}$ damp completely, where $\omega_{n, i}=1.6 \times 10^{-14}\left(n_{2} \zeta_{-17}\right)^{1 / 2} \mathrm{~s}^{-1}$ is the frequency with which a given neutral collides with any ion (Braginskii 1965; Kulsrud \& Pearce 1969). The associated damping length $\lambda_{\min }$ is such that all waves with $\lambda<\lambda_{\min }$ completely damp, while larger wavelength waves can propagate, suffering some damping as they go. From linear theory, the damping length is (see, e.g., CP)

$$
\lambda_{\min }=0.1(B / 12 \mu \mathrm{G}) n_{2} \zeta_{-17}^{-1 / 2} \mathrm{pc},
$$

where $\zeta_{-17}$ is the ionization rate per hydrogen atom measured in units of $10^{-17} \mathrm{~s}^{-1}$ per hydrogen atom, and where we measure the density of the diffuse cloud in units of $10^{2} \mathrm{~cm}^{-3}$. We have taken values for the field and density that seem to best describe the Taurus cloud in order to evaluate $\lambda_{\min }$ in equation (5.5). For clouds that are magnetically subcritical, this damping length is somewhat larger that the Jeans length (Pudritz \& Gomez de Castro 1991). This damping scale is in good agreement with the observed radii of cores in Taurus. Since waves cannot propagate on scales smaller than $\lambda_{\min }$, gas can gather in these quiescent pockets and eventually form cores (CP). Thus wave damping appears to be an excellent means of initiating the formation of the cores we observe in the Taurus cloud. Note that equation (5.6) predicts that as one goes to clouds of higher density, the damping scales become progressively larger.

The time scale for the damping of linear waves is

$$
\tau_{D}=1.0 \times 10^{6} \frac{1}{\left(n_{2} \zeta_{-17}\right)^{1 / 2}}\left(\frac{\lambda}{\lambda_{\min }}\right)^{2} \mathrm{yr},
$$

which is very sensitive to the wavelength.

Let us apply the results (5.1) and (5.6) to the largest scale waves observed in the Taurus cloud. For the long-wavelength wave, $\lambda=16 \mathrm{pc}=160 \lambda_{\min }$ we see that such a disturbance will never damp out for the life of the diffuse Taurus cloud (using eq. [5.7] one gets a time of $10^{10} \mathrm{yr}$ for this wave). This linear theory suggests moreover that since $\lambda_{\min }$ is as small as $0.1 \mathrm{pc}$ in the Taurus cloud, waves of wavelength longer than $0.3 \mathrm{pc}$ will survive for the duration of the cloud's life. Thus waves on the scale 3-5 times larger than the size of the Taurus cores will continue to ring for the life of the cloud once produced in the infall event we have described.

Modes on this scale will not really see the presence of the cores and should not be too adversly dispersed by them by wave reflections. Modes on scales of cores damp in about a cloud free-fall time (a million years). The energy losses on small scales do not pose a great problem for maintaining waves in the cloud since the power dissipated on the scales of cores represents only $1 \%$ of the power in the wave field at the longest 
wavelengths (most of the power must be found at the largest scales if global gravitational collapse is to be staved off). The wave power dissipated on the scale of cores in fact heats such regions to temperatures of $10-20 \mathrm{~K}$ (CP). Thus both the formation of cores and their heating are natural consequences of wave damping in molecular clouds.

\subsection{Waves and Filament-Field Alignments}

It is well known that the magnetic field is not always very well aligned with the filaments in the molecular clouds. This lack of alignment has been interpreted by some authors to imply that the magnetic field on core scales is not dynamically important (Heyer et al. 1987; Strom, Strom, \& Edwards 1988). However, the presence of a wave field in a molecular cloud means that the optical polarization vector changes on some scale $\lambda$ with a period characteristic of Alfvén waves on that scale $T_{w}$. It is important to realize that such a wave field will constantly change the relative alignment of filaments and the magnetic field. Waves of large enough wavelength will not be too affected by reflection from dense filaments, and one ought to see that filament axes and the magnetic field can change their relative alignment over a wave period.

\subsection{The Effects of Weakly Nonlinear Alfvén Waves}

It is clear that the Alfvén wave in the Taurus cloud is of significant amplitude and cannot therefore be regarded as strictly linear. Alfvén waves in linear theory cannot produce density fluctuations. The $\mathrm{H}$ I data suggest however that density fluctuations exist and occur with a spacing that is smaller than that of the Alfvén wave. In this subsection we show that weakly nonlinear waves will indeed induce density fluctuations and can explain the $\mathrm{H}$ I data.

The idea that nonlinear waves induce density fluctuations has been examined by CP and recently Elmegreen (1990). The essence of the phenomenon has been examined in the context of space physics by Spangler (1990). We adapt Spangler's study of a one-dimensional wave to the case of a magnetized, selfgravitating cloud. We present an analytical calculation of this problem in Appendix A where the interested reader can find the details.

These results show that density fluctuations are expected from weakly nonlinear Alfvén type waves. An important consequence of nonlinearity is the appearance of field aligned flow. Suppose that to zeroth order, the cloud field can be considered to have a mean field of strength $B_{0}$ oriented in the $x$-direction. An Alfvén wave perturbs this field in the transverse direction and therefore transverse field components $b_{y}, b_{z}$ arise (to first order). We take this Alfvén wave to have a wavelength $\lambda$. To first order, no density fluctuations are produced, and the cloud is left with its mean density $\rho_{0}$ undisturbed.

Now consider second-order effects. Noting that in a molecular cloud $\beta \ll 1$ and $\alpha \simeq 1$ we find that the density fluctuation produced by weakly nonlinear waves is (from eq. [A8]),

$$
\rho_{2} / \rho_{0} \simeq\left(b_{x}^{2}+b_{y}^{2}\right) / 4 B_{0}^{2} .
$$

To second order, the magnitude of the field-aligned flow is $v_{x}=\left(\rho_{2} / \rho_{0}\right) V_{\mathrm{A}}$ (see eq. [A9]). Thus a good test for such a theory is to look for small amplitude velocities parallel to the field lines.

Our result (see Appendix A, eq. [A8]) also shows that the scale length for density fluctuations is

$$
\lambda_{\rho_{2}}=\lambda / 2 \text {, }
$$

where $\lambda$ represents the wavelength of the first-order linear Alfvén wave. Applying this to the $\mathrm{H}$ I data we conclude that if the main wave has a wavelength of $16 \mathrm{pc}$, then the density fluctuations it produces have a scale length of $8 \mathrm{pc}$. This is in reasonable agreement with the observations of Shuter et al. (1987) who noted that the density enhancements in $\mathrm{H}$ I were separated by $11 \mathrm{pc}$ and that such a spacing is at odds with the velocity data.

\subsection{Nonlinear Effects: Simulations of Single Alfvén Modes}

We have seen that nonlinear Alfvénic waves must induce density fluctuations and motion along the field lines. In a gravitating medium these effects are of great importance since density fluctuations will grow by gravitationally attracting the surrounding fluid. Thus such fluctuations can serve as the "seeds" for the growth of large-scale structure in molecular clouds (see Pudritz 1990 and CP). As a concrete example, such effects from large-scale waves can produce filamentary structures.

In order to demonstrate this gravitational effect of the waves, including the effects of ambipolar diffusion, we ran some numerical experiments using the code described in CP to study exactly what happens when a single mode propagates through an otherwise queiescent magnetized cloud in equilibrium. We ran numerical simulations that allow us to compare cloud models that are stirred or not by a large-scale Alfvén wave. Our initial model is a cold molecular cloud supported by strong isotropic field. An identical initial cloud model is excited by a single Alfvén wave propagating along the $z$-direction in the model as an additional ingredient. We employed the three-dimensional, $N$-body, gas dynamics code ("sticky particles") described in CP to which we refer the reader for the details.

The results of our simulations are in the main given in Pudritz \& Gomez de Castro (1991). In Figure 3 we compare representative spatial slices through the two simulations. The slices are approximately one eighth of the radius of the original cloud in thickness. We stir the models very mildly indeed; in Figure $3 a$ we show a section of a cloud without any wave stirring (supported only by a strong static, isotropic magnetic field) and in Figure $3 b$, we show a slice of a model with a single Alfvén mode whose energy is only $25 \%$ of the gravitational energy density. The Jeans length in this cold cloud calculation is $\lambda_{\mathrm{J}} / R=0.081$ while the wavelength of the single mode we studied is $\lambda / R=0.33$, where $R$ is the initial radius of the cloud. In all calculations the initial state of the cloud is a uniform sphere. Slow contraction of the cloud occurs because of ambipolar diffusion. Our models evolve quasi-statically because our initial state is strongly subcritical.

Figure 3 shows that highly filamentary structure develops very naturally in self-gravitating gas. The reason for this is that any density fluctuations collapse most quickly along the shortest axis. Given that our models have random threedimensional fluctuations (as should a real molecular cloud), one expects that gravity alone will produce a filamented medium. We have always found that such structures develope in a wide variety of models, and we suggest that it is generic in molecular clouds, as indeed the observations strongly show. The action of large-scale waves is not so clearly apparent in the spatial cut in Figure $3 b$, save that more large-scale structures are more highly concentrated and more clearly defined. While the cloud without a wave has some larger scale structure (to be expected simply on the basis of initial fluctuations), the model 


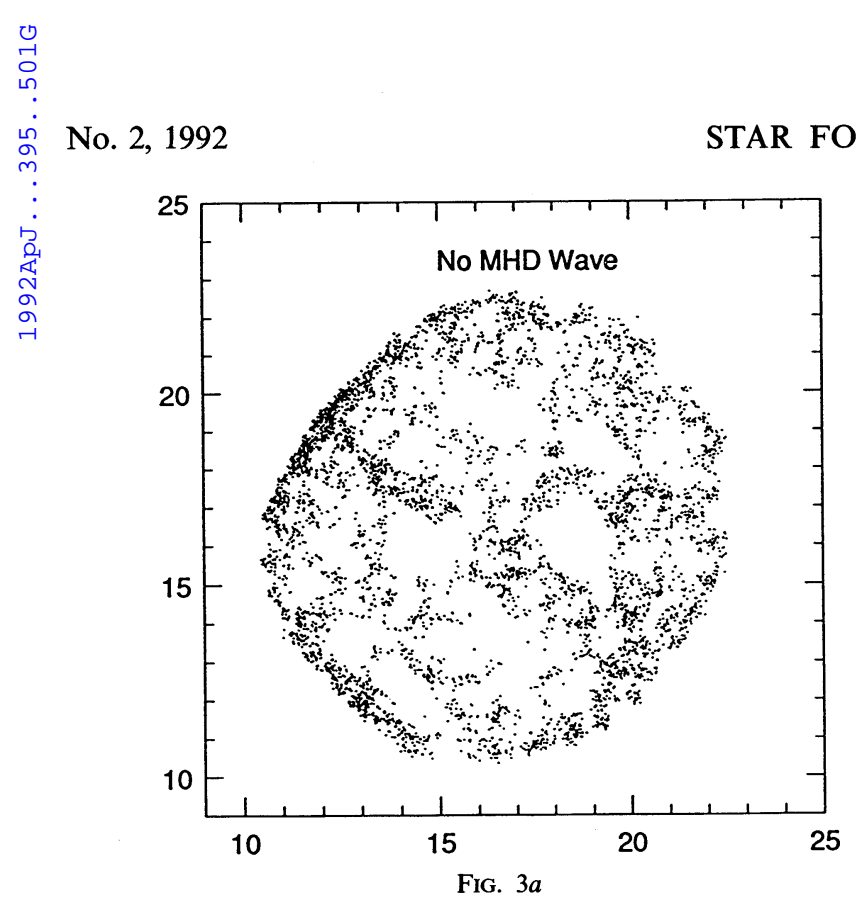

FIG. 3.-Comparison of simulations of magnetically supported, cold molecular clouds with $(a)$ no Alfvén wave and (b) with a single long-wavelength mode. These

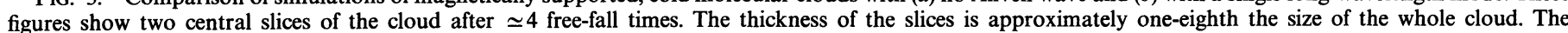
nonmagnetic Jeans length in the cloud is tiny ( 0.081 of the cloud's original radius).

with a wave has much sharper defined filament and massive clump substructure. A mass spectrum analysis of the two models (see Pudritz \& Gomez de Castro 1991) shows indeed that there is more mass tied up in the largest scale structures than in the model with no wave. We emphasize that our perturbation wave is of very low amplitude so that the pictures look similar in spatial structure because of fluctuations in the initial identical cloud models (save for the wave). We furthermore caution that structure on the surfaces of the cloud models is not to be taken too seriously since surface modes will develop rather quickly in these simulations (for the usual reasons).

The simulations show that the cloud breaks up into fragments whose mass is nearly the Jeans mass. As time goes by, agglomeration of the small clumps into more massive ones occurs. However, agglomeration is not the whole picture. By the end of the calculation, the long-mode calculation has significantly more power on the largest scales than does the no-mode simulation. Clearly the long-wavelength wave has swept together gas and created significantly larger scale structure than a model in which long wavelengths are absent. This illustrates the basic point, namely, that waves generate structure.

The effect of agglomeration is to flatten the mass spectrum of the clumps (see Pudritz \& Gomez de Castro 1991 for details). We find that the spectrum appears to turn over at the small mass end. While something like this has been seen observationally, we note that it is an expected feature of clouds which first fragment into small pieces.

We caution the reader that this simulation is a highly idealized experiment and is not meant to be a detailed cloud model. Rather, it is an investigation of some of the simple physics inherent in the wave picture. It should be taken as a demonstration that filamentary structure is an entirely natural development in the self-gravitating and magnetically supported gas of a molecular cloud. A single Alfvénic or magnetosonic wave, or spectrum of waves, can apparently modulate the scales upon which structure formation occurs.

\section{AN MHD SHOCK IN TAURUS}

In this section we argue that there is substantial observational support for the notion of a large-scale shock wave propagating through Taurus. As discussed earlier, one expects the presence of MHD shocks in the Parker instability picture of cloud formation in which motions arise during the main infall phase.

\subsection{Large-Scale MHD Shock in Taurus}

Indications of the presence of a shock wave in Taurus comes from the presence of a velocity shift between the $\mathrm{H}$ I selfabsorption and the rest of the cloud. The $\mathrm{H} \mathrm{I}$ is moving away from the cloud at a speed of $3.8 \mathrm{~km} \mathrm{~s}^{-1}$ which is, to within the experimental uncertainties of the order of the escape velocity from the Taurus cloud, $3.1 \mathrm{~km} \mathrm{~s}^{-1}$. This $\mathrm{H}$ I self-absorbed component is, however, likely to be just gravitationally bound to the cloud because the wavelike ripple observed in the molecular cloud (polarization) is also detected in $\mathrm{H}$ I self-absorption as a velocity wave. Clearly, the self-absorbed component is associated with the cloud magnetic field.

As the magnetic energy of the molecular clouds is comparable to their gravitational self-energy, magnetic fields cannot be ignored, and one necessarily has an MHD shock wave. We are primarily interested in the velocity shift between the $\mathrm{H}$ I selfabsorption and the molecular cloud, so we will concentrate on the effect of the passage of an MHD shock wave through the diffuse $\left(n \leq 10^{3} \mathrm{~cm}^{-3}\right)$ Taurus cloud.

MHD shock waves have been already detected in difiuse molecular clouds along the lines of sight toward $\zeta$ Per, oPer, and $\chi$ Oph (Draine \& Katz 1986a, hereafter DK). For the physical conditions in these clouds and typical shock speeds of about $10 \mathrm{~km} \mathrm{~s}^{-1}$ the shock is C-type, implying that all the flow variables vary continuously through the shock transition (Draine 1980; Draine \& Katz 1986b). The observational signatures of the shock wave passage can be summarized as follows: (1) Two narrow components at different velocities are detected in optical resonance lines as $\mathrm{Ca}$ II or $\mathrm{Na}$ I. The velocities corre- 
spond to the preshock and postshock velocity of the gas; (2) The postshock velocities of the various molecular species are not the same but depend on the charge and formation process in a way that agrees with $\mathrm{C}$-shock predictions; (3) $\mathrm{CH}^{+}$ emission is usually strong.

Evidence for a shock is suggested in the $\mathrm{H}_{\mathrm{I}}$ data from the cloud, and more reliably, from the optical absorption data. Two components are detected in $\mathrm{H}$ I self-absorption in Taurus. One is centered at $\simeq 6.5 \mathrm{~km} \mathrm{~s}^{-1}$ and is essentially associated with the molecular cloud. This component traces the region where the cold $\mathrm{H}$ I condenses and $\mathrm{H}_{2}$ is produced. The cold $\mathrm{H}_{\mathrm{I}}$ is seen in absorption against the hot background $\mathrm{H} \mathrm{I}$ emission. The other self-absorption component has a systemic LSR velocity of $2.7 \mathrm{~km} \mathrm{~s}^{-1}$ and a column density 3 times smaller (Sancisi \& Wesselius 1970).

The optical absorption-line data show two components to be present, which may be summarized as

$$
\begin{array}{ll}
\text { Component 1: } & V_{*, 1}=7.4 \mathrm{~km} \mathrm{~s}^{-1}, \quad N \simeq 80 \mathrm{~cm}^{-3}, \\
\text { Component 2: } & V_{*, 2}=3.4 \mathrm{~km} \mathrm{~s}^{-1}, \quad N \simeq 25 \mathrm{~cm}^{-3},
\end{array}
$$

where $V_{*, 1}$ and $V_{*, 2}$ are LSR velocities. These two components have also been detected along the line of sight to $\zeta$ Per, located at the North-West end of the Taurus Complex $\left(\alpha=3^{\text {h }} 51^{\mathrm{m}}\right.$ and $\delta=31^{\circ} 44^{\prime}$ ). The dominant component, at an LSR velocity $V_{*, 1}=7.4 \mathrm{~km} \mathrm{~s}^{-1}$, is detected in $\mathrm{Na}$ I (Hobbs 1969), Ca II (Marshall \& Hobbs 1972), K I (Hobbs 1974), and Ti II (Hobbs 1979). This is the same velocity as the molecular gas near this position (see $\$ 2.2$ ). The weaker component is moving at a velocity $V_{*, 2}=3.4 \mathrm{~km} \mathrm{~s}^{-1}$. The slight differences between the optical and $\mathrm{H}$ I absorption data are likely due to the velocity gradient from the NE to SW in the cloud. The optical data originate from measurements on a star located in the NW end of the cloud, where the radial velocities are slightly larger than the cloud center. The H I data, however, sample the more of the cloud, and therefore the average absorption feature is at a smaller velocity. We rely most upon the stellar data following the work of DK.

In DK's work the component at $7.4 \mathrm{~km} \mathrm{~s}^{-1}$ is assumed to trace the denser postshock gas. They are able to reproduce the molecular line velocities and intensities with the passage of a shock wave moving at an LSR shock speed $V_{\text {sh }}=6.7 \mathrm{~km} \mathrm{~s}^{-1}$ and making an angle of $46^{\circ}$ with the line of sight. The velocity of the shock wave is essentially fixed by the lines intensities and the inclination by the equation

$$
V_{*, 1}=3.4+V_{\text {sh }} \cos (\theta),
$$

where $V_{*, 1}$ represents the radial postshock speed of the neutrals. This equation reflects the important property of the C-type MHD shocks, that the velocity of the postshock gas with respect to the preshock is approximately the same as the velocity of shock front (see, e.g., DK).

The geometry of the shock as inferred from DK is sketched in Figure 4. The higher density component represents the compressed postshock gas moving at radial speeds of $7.4 \mathrm{~km} \mathrm{~s}^{-1}$, while the lower density preshock gas is moving at $3.4 \mathrm{~km} \mathrm{~s}^{-1}$. The shock front propagates at an angle of $46^{\circ}$ respect to the line of sight in order to reproduce the projected velocity difference. This geometry can also apply to the scale of the whole Taurus cloud. In fact the inclination angle is, to within the uncertainties, close to the inclination of the motion of the Taurus cloud with respect to the line of sight, as pointed out in equation (2.1). Moreover, the $\mathrm{H}$ I column density of the post- shock gas, $\log _{10} N_{\mathrm{HI}}\left(\mathrm{cm}^{-2}\right) \simeq 18.8$ (Wilson \& Minn 1977) is of the same order than the inferred from DK's model, $\log _{10} N(\mathrm{H} \mathrm{I})\left(\mathrm{cm}^{-2}\right)=18.0$.

The time scale for the shock wave passage through the region is comparable to the star formation time scale. In fact, the projected size of Taurus in the sky is $\simeq 17 \mathrm{pc}$ and takes $3.6 \times 10^{6} \mathrm{yr}$ for the $6.7 \mathrm{~km} \mathrm{~s}^{-1}$ shock wave to cross the cloud. Therefore, if the shock wave affects the star formation rate, by compressing cloud cores as an example, we might expect some differences in the star formation rates in pre- and postshocked regions of the cloud. We examine this question in the following section.

In order to better understand how much of the Taurus cloud has been affected by the shock, it is important to have some three-dimensional picture of the cloud. There is good evidence that it does not lie entirely in the plane of the sky, but rather makes a large angle with it (as has been proposed by many authors). Kleiner \& Dickman (1984) suggested that Taurus complex lies at "a dusty tongue of material protruding inward toward the Sun," and inclined at $\simeq 30^{\circ}$ with respect to the line of sight. Note that this direction is perpendicular to the motion of the clouds and parallel to the shock front as described in Figure 4 (also compare with Fig. 2 from Paresce 1984). Therefore it is likely that the shock is advancing over the whole complex at the same time. We suggest that the filaments are at different distances as sketched in Figure 4. In fact, the distance estimates to Taurus vary from the $100 \mathrm{pc}$ of Heiles Cloud 2 (Cernicharo \& Guelin 1987) to the 140 pc generally adopted distance (UT).

\subsection{Caveats}

The shock properties we have discussed are derived from the assumption that the component I moving at $V_{*, 1}=7.4 \mathrm{~km}$ $\mathrm{s}^{-1}$, represents the postshock gas. This is based on the larger column density of this component, assumed to represent a larger particle density. This assumption is at risk if the diffuse Taurus cloud has other large-scale inhomogeneities in the $\mathrm{H}$ I other than those generated by the passage of a $\mathrm{C}$ shock.

The exact geometry of the shock is also difficult to pin down without more work. The main infall phase which formed the cloud leaves the cloud with a motion in the plane of Gould's Belt as we have earlier argued. The resulting cloud will shock with the surrounding interstellar medium. Smaller amounts of gas will continue to rain down upon the cloud, however, as the buoyant magnetic loop continues to rise. This continued small amount of infall might explain the existence of a population of higher speed H I clouds deduced by Kulkarni \& Fich (1985) which have a cloud-cloud velocity dispersion of $35 \mathrm{~km} \mathrm{~s}^{-1}$. The possible small amount of continued infall, plus the superAlfvénic motion of the cloud through undisturbed ISM, may make for a fairly complicated shock; that is beyond the purpose of this paper to analyze in detail. Both continued infall (in small amounts) and bulk cloud motion are associated with shock fronts.

\section{CONSEQUENCES FOR STAR FORMATION IN TAURUS}

\subsection{The Core Mass Function}

It is by now well established that there is a close relation between the $\mathrm{NH}_{3}$ cores and young embedded infrared sources (Beichman et al. 1986; Benson \& Myers 1989). The $\mathrm{NH}_{3}$ cores in Taurus are concentrated at the SE end of the filaments, 


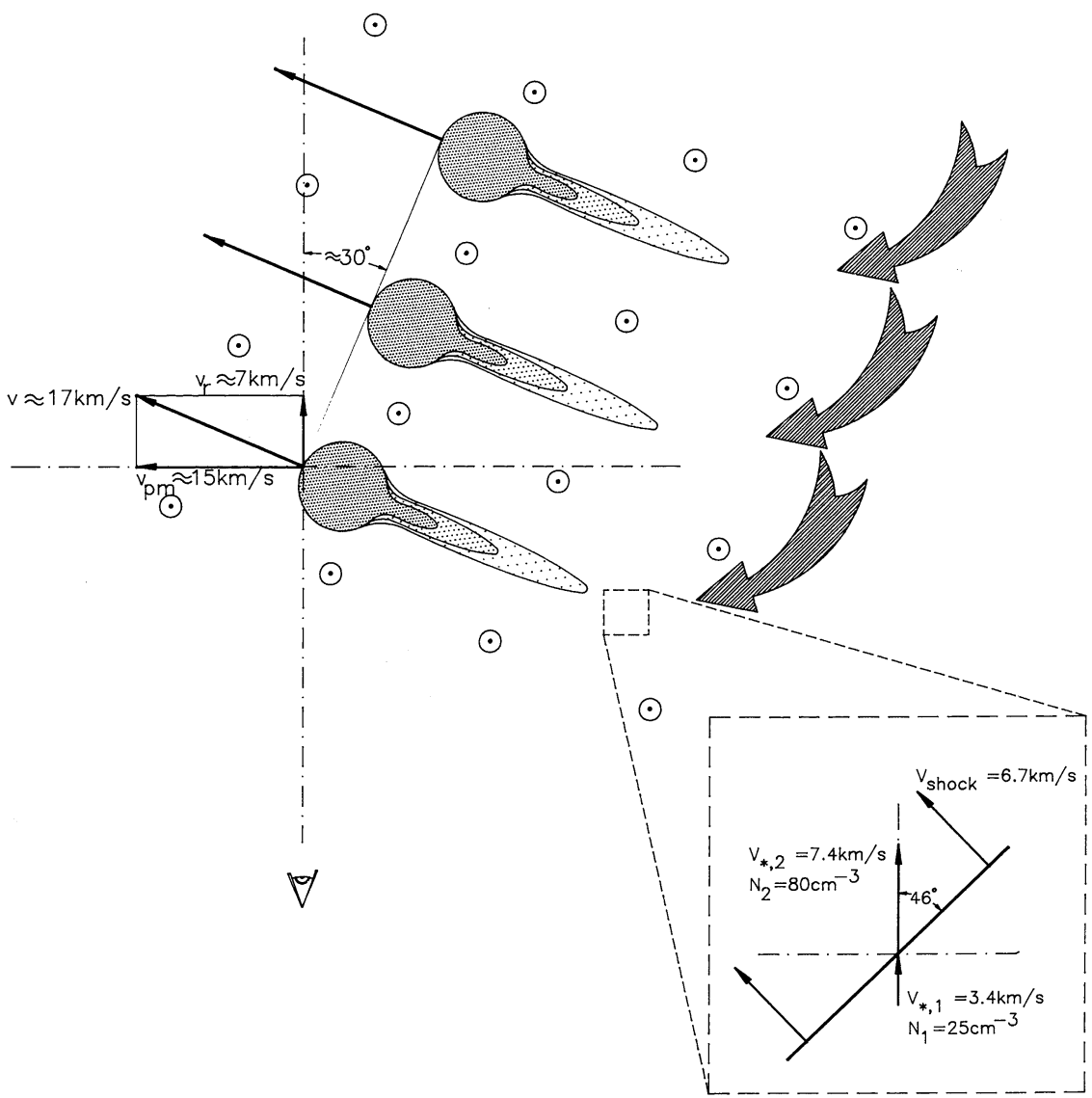

Fig. 4. - Sketch of the shock geometry in Taurus seen from above the Galactic plane. The direction to the observer is indicated. The Taurus cloud is represented as a collection of filaments located at different distances from the Sun and moving with a mean velocity of $17 \mathrm{~km} \mathrm{~s} \mathrm{~s}^{-1}$. The components in the line of sight and in the plane of the sky are indicated. The magnetic field is roughly perpendicular to the Galactic plane. Some material falling back onto the Gould's Belt is indicated by the

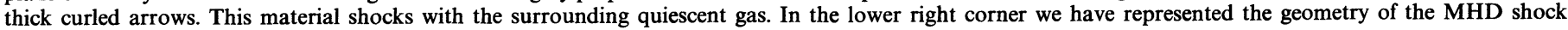
occurring in Taurus as inferred from DK's study. Only radial velocities of the pre and postshock gas are indicated.

where also most of the PMS stars and PMS IRAS sources are located. They are most likely prolate structures with mean radii lying in the range $0.06-0.15$ pc (Myers et al. 1991).

The masses of the Taurus cores have been measured using different tracers $\left(\mathrm{C}^{18} \mathrm{O}, \mathrm{CS}\right.$, and $\mathrm{NH}_{3}$ ) and procedures (see Myers et al. 1983; Myers \& Benson 1983; Benson \& Myers 1989; Fuller 1989). The most reliable estimates are those based on $\mathrm{NH}_{3}$ data computed by Benson \& Myers (1989). Most of the cores, 10 out of 14 , have masses smaller than $5 M_{\odot}$; the other four cores, TMC-2, L1536, TMC-1A, and L1527, are substantially more massive, $16,33,13$, and $15 M_{\odot}$, respectively. These massive cores are not uniformly distributed, but concentrated in the SE end of the cloud.

We have used these masses to obtain the cumulative mass function of the cores $\left(N_{\text {core }}\right)$. The mass spectrum of cores in molecular clouds has been modeled as a power law by many authors,

$$
\frac{d N_{\text {core }}(m)}{d m} \propto m^{-\alpha}
$$

We shall henceforth call this function the Core Mass Function (CMF), representing as it does the number of cores with mass $m$ in the interval of core mass from $m$ to $m+d m$.

Clearly a sample of only 14 cores can at best give only a rough idea of the distribution. Nonetheless, we find a good power-law fit to the data, which is shown in Figure 5, with a slope

$$
\alpha=1.37 \pm 0.05 \text {. }
$$

This value for the index in Taurus is similar to that for the

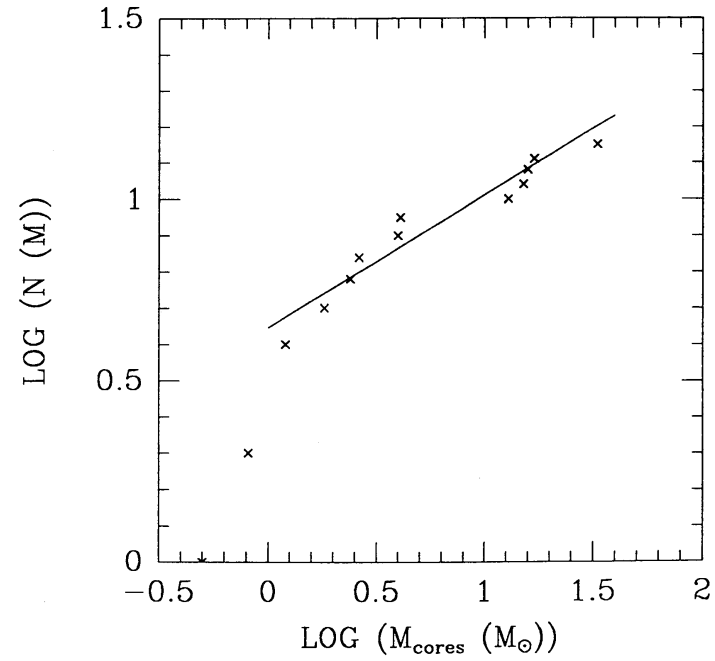

Fig. 5.-The core mass function (CMF) $d N_{\text {core }} / d m$ of the Taurus cloud $\mathrm{NH}_{3}$ cores. The straight line shows the power-law fit to the mass function given in the text. 
Rosette Nebula ( $\alpha \simeq 1.5$; Blitz 1987) but slightly different from that of $\rho$ Oph where $\alpha \simeq 1.1$ (Loren 1989a, b). One should realize that the data plotted in Figure 5 suffer from the obervational selection effect that it is difficult to find very low mass cores.

There is a striking difference between the CMF and the initial mass function (IMF) of young stars in the Taurus cloud. The IMF for Taurus determined by Cohen \& Kuhi (1979) is nearly the Salpeter function, that is,

$$
\frac{d N_{*}\left(m_{*}\right)}{d m_{*}} \propto m_{*}^{-2.5} .
$$

A comparison of the CMF and IMF for Taurus implies that the cores are far more dominated by massive clumps, while the stars are dominated by the lower mass members of the distribution. Clearly there is an important piece of physics that dictates how one goes from cores to stars. If one applies the view that there is one star per core, then there is no explanation of the data other than for some mysterious reason, massive cores are much more inefficient at forming stars! Recent IR camera observations carried out by Lada et al. (1991) in Orion show clusters with, at least, 300 stars being formed in the interior of dense molecular cores. A cluster mode, as suggested by these observations, could be universal. In this case, a second picture is possible. That is simply that the massive cores must produce many stars. Since most of the core mass is tied up in the most massive cores, it seems plausible that most of the star formation, even in the Taurus cloud, is likely to be in the form of small associations of stars within the more massive cores. While any such cluster in the most massive Taurus cloud would only contain several stars, this does not change the basic physical idea that star formation in molecular clouds is dominated by a cluster-producing process.

\subsection{Star Formation}

Given that a shock wave propagates through Taurus, are we likely to expect strong effects on the details of star formation? Could we detect noticeable differences in the type of star formation that occurs depending on the dynamical state of the cloud gas? An an example, the two Taurus groups have a relative velocity difference of $20-15=5 \mathrm{~km} \mathrm{~s}^{-1}$. Did star formation proceed noticeably differently in these groups?

In examining these issues, we note that there is no completely foolproof method of comparing the young stellar populations in different parts of the cloud. One approach that has been used in the literature is to assume that younger PMS stars are more active than the more evolved TTS so that the strength of the $\mathrm{H} \alpha$ emission is a reliable indicator of the degree of activity (Herbig 1978; Walter 1987). If activity measured in this line roughly correlates with the youth of a young stellar object, then one can try to compare the luminosity functions of young stellar objects in different clumps within the cloud.

We have therefore selected all the TTS in Taurus whose $\mathrm{H} \alpha$ equivalent width is known. The sample consists of stars taken from the Herbig and Bell Catalogue (HBC) plus the sources found recently by Kenyon et al. (1990) and Hartmann et al. (1991).

The cumulative distribution functions are shown in Figure 6. The distributions contain the 30 PMS stars in region I and the 59 of the 65 PMS located in region II whose $W(\mathrm{H} \alpha)$ is known. We see that these two distributions are very similar. In order to make this quantitative, we employ the Kolmogorov-Smirnov test. The test shows that both distributions are not different to

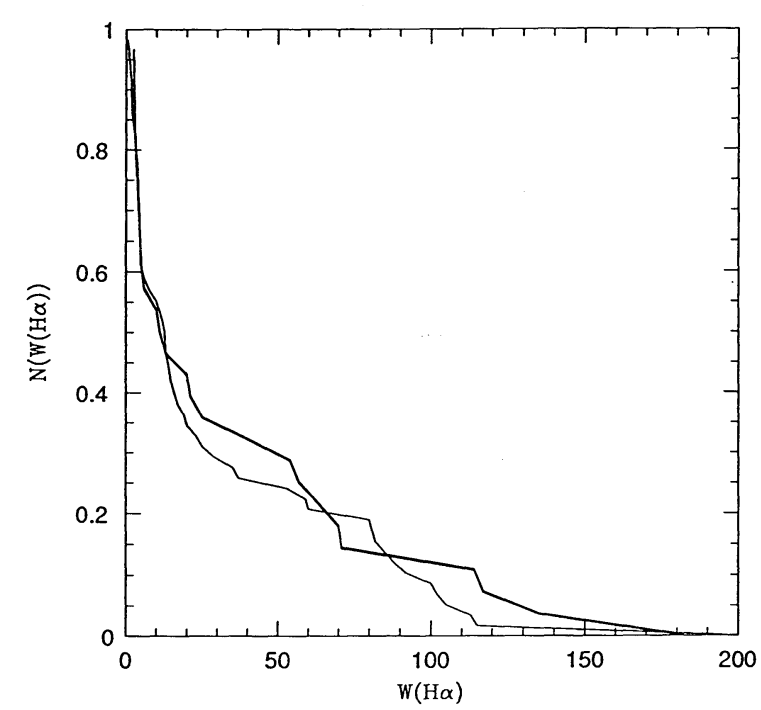

Fig. 6.-Cumulative $\mathrm{H} \alpha$ distribution function for the stars in group I (thick line) and group II (thin line)

a significance level of 0.9994 . Therefore, if $\mathrm{H} \alpha$ activity even roughly correlates with the age of young stellar objects, then the stellar populations in the two groups are approximately the same. This points out that whatever the mechanism regulating the star formation in Taurus is, it is acting over the whole complex and does not generate significant differences in "evolution times" and mass functions inside it.

This conclusion agrees with the independent results found by Cernicharo, Bachiller, \& Duvert (1986), for the ages of the dark clouds that constitute the Taurus Complex. They studied the ratios of the cyanopolyynes (complex molecules whose general formula is $\mathrm{HC}_{2 n+1} \mathrm{~N}$ ) and that can be used as a tracer of cloud age (Stahler 1984). They concluded that the ratios are essentially the same in the seven regions of the Complex studied by them (L1544, L1521, L1495, HCL2-B, TMC1-C, TMC-1, and TMC2), and that therefore if the main path to the cyanopolyne formation is sequential, that all the Taurus clouds should have the same age.

These results for the ages of the two groups are readily understood if we remember that the current massive starforming cores in Taurus are likely to have been recently shocked as discussed earlier in the paper. If this is true, the $M H D$ shock wave may be providing the overpressure that has started copious star formation in these cores.

\section{CONCLUSIONS}

We have employed data sets that include stellar proper motions, gas velocities such as $\mathrm{H}$ I and $\mathrm{CO}$, and optical polarization in order to derive a global view of gas dynamics and star formation in the Taurus cloud. Assuming only that gas and stars are well coupled, we can determine the velocity of the Taurus complex through the ISM. The bulk motion of the cloud, inferred to be $17 \mathrm{~km} \mathrm{~s}^{-1}$, is generated by the infall of material onto the Gould Belt plane as a consequence of Parker-Jeans instability in the Local ISM. The bulk motion of the cloud in Gould's Belt is super-Alfvenic with respect to the surrounding quiescent ISM and shocks are expected which may be detected in the $\mathrm{H}$ I self-absorption. 
Among our most important conclusions are the following:

1. The Taurus cloud is a set of cometary-shaped filaments moving with an inclination of $67^{\circ}$ with respect to the line of sight. The most striking aspect of the stellar data is that T-Tauri stars appear to move roughly parallel to the filaments in the same direction as the Gould's Belt members.

2. The total cloud field is inferred to be about $12 \mu \mathrm{G}$ and is largely in the plane of the sky. Optical polarization data suggest the field contains a wave with wavelength $\simeq 17 \mathrm{pc}$. The Alfvén speed in the cloud is $1.5 \mathrm{~km} \mathrm{~s}^{-1}$, and the wave has a period of $1.1 \times 10^{7} \mathrm{yr}$.

3. The MHD shock direction is nearly perpendicular to the direction of the mean magnetic field in the cloud. It is inferred to have a speed of $\sim 6.7 \mathrm{~km} \mathrm{~s}^{-1}$ through the cloud. $\mathrm{H}$ I absorption data show that this component moves at a speed that is $3.8 \mathrm{~km} \mathrm{~s}^{-1}$ slower than the $\mathrm{CO}$ which can be explained in terms of the expected velocity differences between pre- and postshock gas within a $\mathrm{C}$ shock (DK). More observations of background stars are required to confirm the existence of the shock acting over the whole cloud.

4. The $\mathrm{H}$ I data indicate the presence of a wavelike velocity structure with wavelength $\lambda_{\mathrm{HI}} \simeq 16 \mathrm{pc}$, which is nearly identical to that inferred in the optical polarization data. Large-scale waves with wavelengths comparable to cloud size might be generated during the main infall phase of gas disrupted by the Parker instability. The presence of large-scale waves in the magnetic field of the cloud implies that a variety of apparent alignments between the magnetic field and the long axes of the gas filaments are possible. In the dynamic environment of a cloud, these orientations change on a $10^{6}-10^{7}$ yr period. The largest scale waves in a cloud persist over the life of the cloud without severe damping. They can be thought of as the ringing echoes of the main infall event.

5. The $\mathrm{NH}_{3}$ cores in Taurus have a mass function $d N /$ $d m \simeq m^{-1.4}$. The fact that the core mass function is steeper than the slope of the IMF in general implies that the formation of a cluster of stars within massive cores is to be expected.
6. The theory of weakly nonlinear Alfvén waves predicts that such waves must produce density fluctuations. If the nearly linear wave has a wavelength of $\lambda$, theory predicts that density fluctuations have the period $\lambda / 2$. We suggest this as the explanation of density enhancements in the $\mathrm{H}$ I data that are spaced shorter than that of the velocity wave. Nonlinear simulations that incorporate the effects of ambipolar diffusion confirm that a single mode produces large-scale density fluctuations. These simulations also show that there is intensive agglomeration of small-scale clumps in a cloud (which are of order of a Jeans length in size) to form more massive clumps. This is likely to be important in understanding the genesis of the core mass function.

7. Finally, it has been shown that the star formation in Taurus is intimately linked to the dynamics of the Gould's Belt. The origin of the molecular clouds is generally assumed to be connected with the onset of an odd-mode, Jeans-Parker instability. We have shown that energy which drives the shock and cloud stirring arises naturally from the gravitational energy released as uplifted gas falls back forming a shocked gas layer (Gould's Belt) that is inclined to the Galactic plane. The component of the gas momentum that is parallel to the layer is responsible for the observed motion of the Taurus cloud in the plane of Gould's Belt.

We are indebted to Alyssa Goodman for her detailed and insightful remarks as the referee. We also wish to thank Phil Myers and Mark Heyer for stimulating discussions and remarks, and Federico de Isidro for his help with the CAD programs that have been used in this project. This research was supported by a grant from NSERC of Canada. A. I. G. d. C. gratefully acknowledges support from the Ministerio de Educación y Ciencia of Spain and NSERC of Canada for the International Postdoctoral Fellowships enjoyed in 1989-1990 and 1990-1991, respectively. R. E. P. thanks the Canadian Institute for Theoretical Astrophysics (CITA) for the award of a Reinhardt Fellowship that allowed some of this work to be completed.

\section{APPENDIX}

\section{NONLINEAR ALFVÉN WAVES}

Following Spangler (1990), we assume that a uniform mean field of strength $B_{0}$ runs parallel to the $x$-axis and that all physical variables are functions of time $t$ and $x$. The condition $\nabla \cdot \boldsymbol{B}=0$ implies that the total field can be written as

$$
\boldsymbol{B}=B_{0} \hat{\boldsymbol{x}}+b_{y} \hat{\boldsymbol{y}}+b_{z} \hat{z} .
$$

We write the velocity field as $\boldsymbol{v}=v_{x} \hat{\boldsymbol{x}}+v_{y} \hat{\boldsymbol{y}}+v_{z} \hat{z}$. The induction equation governing the evolution of the magnetic field then reduces to two nontrivial equations;

$$
\begin{aligned}
& \frac{\partial b_{y}}{\partial t}=B_{0} \frac{\partial v_{y}}{\partial x}-\frac{\partial\left(v_{x} b_{y}\right)}{\partial x}, \\
& \frac{\partial b_{z}}{\partial t}=B_{0} \frac{\partial v_{z}}{\partial x}-\frac{\partial\left(v_{x} b_{z}\right)}{\partial x} .
\end{aligned}
$$

As Spangler emphasizes, it is the presence of a field-aligned velocity $v_{x}$ that brings about the non-Alfvénic character of the waves. The equations of motion for $v_{y}$ and $v_{z}$ are

$$
\begin{aligned}
& \frac{\partial v_{y}}{\partial t}+v_{x} \frac{\partial v_{y}}{\partial x}=\frac{B_{0}}{4 \pi \rho} \frac{\partial b_{y}}{\partial x} \\
& \frac{\partial v_{z}}{\partial t}+v_{x} \frac{\partial v_{z}}{\partial x}=\frac{B_{0}}{4 \pi \rho} \frac{\partial b_{z}}{\partial x}
\end{aligned}
$$


Thus in the absence of field-aligned flow, these equations reduce to a pair of Alfvén waves. Nonlinear waves must therefore be associated with some amount of field-aligned flow.

The equation of motion for $v_{x}$ is

$$
\frac{\partial v_{x}}{\partial t}=-\frac{1}{8 \pi \rho} \frac{\partial^{2}\left(b_{y}^{2}+b_{z}^{2}\right)}{\partial x^{2}}-\frac{c_{s}^{2}}{\rho} \frac{\partial^{2} \rho}{\partial x^{2}}-\frac{\partial \phi}{\partial x}
$$

where $\phi$ is the gravitational potential and the pressure is $p=\rho c_{s}^{2}$. In addition to these relations one requires the continuity equation

$$
\frac{\partial \rho}{\partial t}+\frac{\partial\left(\rho v_{x}\right)}{\partial x}=0
$$

as well as the Poisson equation.

Spangler employs an elegant, physically motivated ordering scheme (Hollweg 1971) which avoids the tedious calculation of a reductive perturbative method. To zeroth order we have a static mean field $B_{0}$. To first order, the field is described by a propagating Alfvén wave with components $b_{y}$ and $b_{z}$ with associated first-order velocities $v_{y}$ and $v_{z}$. In this ordering, the leading order for $v_{x}$ is second order. The density is taken to have a constant zeroth-order part $\rho_{0}$, but since an Alfvén wave does not induce density fluctuations, the leading correction is second order $\rho_{2}$. Further correction to $b_{y}, b_{z}, v_{y}$ and $v_{z}$ are all third order.

Employing this physics, it is easy to confirm that to first order, equations (A2) and (A3) give Alfvén wave solutions for the transverse speeds and fields

$$
\begin{aligned}
& b_{y}=\hat{b}_{y} e^{i(k x-\omega t)}, \\
& b_{z}=\hat{b}_{z} e^{i(k x-\omega t)},
\end{aligned}
$$

etc., where the frequency of the wave is given by the dispersion relation for Alfvén waves

$$
\omega=k V_{\mathrm{A}}, \quad V_{\mathrm{A}} \equiv \frac{B_{0}}{4 \pi \rho_{0}} .
$$

To second order one solves for $v_{x}$ and $\rho_{2}$ using equations (A4) and (A5), as well as the Poisson equation to eliminate the gravitational potential; $\partial^{2} \phi_{2} / \partial x^{2}=4 \pi G \rho_{2}$. The solutions are

$$
\begin{aligned}
& \rho_{2}=\hat{\rho}_{2} e^{i(2 k x-2 \omega t)}, \\
& v_{x}=\hat{v}_{x} e^{i(2 k x-2 \omega t)},
\end{aligned}
$$

where

$$
\begin{gathered}
\hat{v}_{x}=\frac{V_{\mathrm{A}}}{\rho_{0}} \hat{\rho}_{2}, \\
\frac{\hat{\rho}_{2}}{\rho_{0}}=\frac{\left(\hat{b}_{y}\right)^{2}+\left(\hat{b}_{z}\right)^{2}}{2 B_{0}^{2}} \frac{1}{(1-\beta+\alpha)} .
\end{gathered}
$$

The parameters $\alpha$ and $\beta$ are the ratio of gravitational to mean magnetic field energy density, and thermal to mean magnetic field energy density respectively:

$$
\begin{gathered}
\alpha \equiv \frac{4 \pi G \rho_{0}}{k^{2} V_{\mathrm{A}}^{2}}, \\
\beta \equiv \frac{c_{s}^{2}}{V_{\mathrm{A}}^{2}} .
\end{gathered}
$$

Our result differs from Spangler's analysis only through the additional gravitational term.

\section{REFERENCES}

Arons, J., \& Max, C. E. 1975, ApJ, 196, L77

Barnard, E. E. 1927, in Carnegie Institute of Washington Publ. No. 247, I

Beichman, C. A., Myers, P. C., Emerson, J. P., Harris, S., Mathieu, R., Benson,

P. J., \& Jennings, R. E. 1986, ApJ, 307, 337

Benson, P. J., \& Myers, P. C. 1989, ApJS, 71, 89

Blitz, L. 1987, in Physical Processes in Interstellar Clouds, ed. G. E. Morfill \& M. Scholer (Dordrecht: Kluwer), 35

Blitz, L., \& Shu, F. 1980, ApJ, 238, 148

Bonazzola, S., Falgarone, E., Heyvaerts, J., Perault, M., \& Puget, J. L. 1987, A\&A, 172, 293

Braginskii, S. I. 1965, Rev. Plasma Phys., 1, 205

Carlberg, R. G., \& Pudritz, R. E. 1990, MNRAS, 247, 353 (CP)

Cernicharo, J., Bachiller, R., \& Duvert, G. 1985, A\&A, 149, 273 1986, A\&A, 160, 181

Cernicharo, J., Castets, G., Duvert, G., \& Guilloteau, S. 1984b, A\&A, 139, L13
Cernicharo, J., \& Guelin, M. 1987, A\&A, 176, 299

Cernicharo, J., Guélin, M., \& Askne, J. 1984a, A\&A, 138, 371

Cohen, M., \& Kuhi, L. V. 1979, ApJ, 41, 743

Cox, D. P. 1979, ApJ, 234, 863

Draine, B. T. 1980, ApJ, 241, 1021

Draine, B. T., \& Katz, N. 1986a, ApJ, 310, 392 (DK)

. 1986b, ApJ, 306, 655

Duvert, G., Cernicharo, J., \& Baudry, A. 1986, A\&A, 164, 349

Eggen, O. J. 1986, AJ, 92, 1074

- 1989, Fund. Cosmic Phys., 13, 1

Elmegreen, B. G. 1982, ApJ, 253, 655

. 1990, ApJ, 361, L77

Frogel, J. A., \& Stothers, R. 1977, AJ, 82, 890

Fuller, G. A. 1989, Ph.D. thesis, Univ. California, Berkeley

Goodman, A. A., Bastien, P. C., Myers, P. C., \& Ménard, F. 1990, ApJ, 359, 363 
Goodman, A. A., Crutcher, R. M., Heiles, C., Myers, P. C., \& Troland, T. H. 1989, ApJ, 338, L61

Goodman, A. A., Myers, P. C., Bastien, P., Crutcher, R. M., Heiles, C., Kazés, I., \& Troland, T. H. 1991, in IAU Symp. Galactic and Extragalactic Magnetic Fields, 140, ed. R. Beck, P. P. Kronberg, \& R. Wielebinsky (Dordrecht: Kluwer), 319

Hartmann, L., Hewett, R., Stahler, S., \& Mathieu, R. D. 1986, ApJ, 309, 275 Hartmann, L., Jones, B. F., Stauffer, J. R., \& Kenyon, S. J. 1991, AJ, 101, 1050 Heiles, C. 1988, ApJ, 324, 321

Herbig, G. H. 1978, in Problems of Physics and Evolution of the Universe (Yervan: Academy of Sciences of the Armenian SSR), 171

Heyer, M. H., Vrba, F. J., Snell, R. L., Schloerb, F. P., Strom, S. E., Goldsmith, P. F., \& Strom, K. M. 1987, ApJ, 321, 855

Hobbs, L. M. 1969, ApJ, 157, 135

.1974, ApJ, 191, 381 1979 ApJ 229 , L129

Hollweg J. V. 1971, J. Geophys. Res., 76, 5155

Horiuchi, T., Matsumoto, R., Hanawa, T., \& Shibata, K. 1988, PASJ, 40, 147

Hsu, J. C. 1984, Ph.D. thesis, Univ. Texas, Austin

Jones, B. F., \& Herbig, G. H. 1979, AJ, 84, 1872 (JH)

Kenyon, S. J., Hartmann, L. W., Strom, K. M., \& Strom, S. E. 1990, AJ, 99, 869

Kleiner, S. C., \& Dickman, R. L. 1984, ApJ, 286, 256

Kulkarni, S., \& Fich, M. 1985, ApJ, 289, 792

Kulkarni, S. R., \& Heiles, C. 1988, in Galactic and Extragalactic Radio Astronomy, ed. G. L. Verschuur \& K. I. Kellermann (New York: Springer), 95

Kulsrud, R., \& Pearce, W. P. 1969, ApJ, 156, 445

Lada, E., DePoy, D. L., Evans, N. J., \& Gatley, I. 1991, ApJ, 371, 171

Lesh, J. R. 1968, ApJS, 151, 371

Linblad, P. O. 1980, Mitt. Astron. Ges., 48, 15

Loren, R. B. 1989a, ApJ, 338, 902 1989b, ApJ, 338, 925

Marshall, L. A., \& Hobbs, L. M. 1972, ApJ, 248, 1029

Mathewson, D. S. 1968, ApJ, 153, L47

Mathewson, D. S., \& Ford, V. L. 1970, MmRAS, 74, 139

Matsumoto, R., Horiuchi, T., Hanawa, T., \& Shibata, K. 1988, PASJ, 40, 171
Moneti, A., Pipher, J. L., Helfer, H. L., McMillan, R. S., \& Perry, M. L. 1985, ApJ, 282, 508

Myers, P. C., \& Benson, P. J. 1983, ApJ, 266, 309

Myers, P. C., Fuller, G. A., Goodman, A. A., \& Benson, P. J. 1991, ApJ, 376 561

Myers, P. C., \& Goodman, A. 1988, ApJ, 32, L27 1991, ApJ, 373, 509

Myers, P. C., Linke, R. A., \& Benson, P. J. 1983, ApJ, 264, 517

Paresce, F. 1984, AJ, 89, 1022

Pudritz, R. E. 1990, ApJ, 350, 195

Pudritz, R. E., \& Gomez de Castro, A. I. 1991, IAU Symp. 147, Fragmentation of Molecular Clouds and Star Formation, ed. E. Falgarone \& G. Duvert (Dordrecht: Kluwer), 317

Sancisi, R., \& Wesselius, P.D. 1970, A\&A, 7, 341

Scalo, J. 1990, in Physical Processes in Fragmentation and Star Formation, ed R. Capuzzo-Dolcetta, C. Chiosi, \& A. Di Fazio (Dordrecht: Kluwer), 151

Schneider, S., \& Elmegreen, B. G. 1979, ApJS, 41, 87

Schwartz, R. 1977, ApJS, 35, 161

Shuter, W., \& Dickman, R. 1989, in Molecular Clouds in the Milky Way and External Galaxies, ed. R. Dickman et al. (Berlin: Springer), 231

Shuter, W. L., Dickman, R. L. \& Klatt, C. 1987, ApJ, 322, L103

Spangler, S. R. 1990, Phys. Fluids B, 2, 407

Stahler, S. W. 1984, ApJ, 281, 209

Stothers, R., \& Frogel, J. A. 1974, AJ, 79, 456

Strom, S. E., Strom, K. M., \& Edwards, S. 1988, Galactic and Extragalactic Star Formation, ed. R. Pudritz \& M. Fich (NATO ASI Series) (Washington GPO), 53

Taylor, D. K., Dickman, R. L., \& Scoville, N. Z. 1987, ApJ, 315, 104

Troland, T. H., \& Heiles, C. 1982, ApJ, 252, 179

Ungerechts, H., \& Thaddeus, P. 1987, ApJS, 63, 645 (UT)

Vardanyan, R. A., \& Eritsyan, M. A. 1984, Astrofizika, 19, 719

Verschuur, G. L. 1971, ApJ, 165, 651

Vrba, F. J., Strom, S. E., \& Strom, K. M. 1976, AJ, 81, 958

Walter, F. M. 1987, PASP, 99, 31

Westin, T. N. G. 1987, A\&AS, 60, 99

Wilson, T. L., \& Minn, Y. K. 1977, A\&A, 54, 933 